\title{
Hepato-splenic candidiasis confirmed following splenectomy: a case report
}

\author{
Alexandre Peinoit' ${ }^{1}$ Elie Cousin ${ }^{1}$, Martine Escoffre-Barbe ${ }^{1}$, Marc Bernard ${ }^{1}$, François \\ Benezit ${ }^{2}$, Thierry Lamy ${ }^{1}$ \\ 1Department of Hematology, University Hospital of Rennes, Rennes, France; \\ ${ }^{2}$ Department of Infectious Diseases, University Hospital of Rennes, Rennes, France
}

Article received 16 September, 2021; accepted 10 November, 2021

\section{SUMMARY}

We report a case of severe refractory hepato-splenic candidiasis confirmed following splenectomy in an immunocompromised 54-year-old woman treated for acute lymphoblastic leukemia. Liver biopsies did not contribute to identifying the etiology of the multiple hepato-splenic lesions. In view of the lack of improvement after several lines of antifungal treatments, a splenectomy was performed and confirmed splenic candidiasis. PET/CT scan was used to monitor the evolution of hepato-splenic hypermetabolism. Splenectomy can be envisaged to establish fungal infection in immuno-compromised patients, to exclude relapse, and to guide the choice of antifungal agents.

Keywords: Hepato-splenic candidiasis, splenectomy, acute lymphoblastic leukemia, immuno-compromised.

\section{INTRODUCTION}

$\mathrm{H}$ epato-splenic candidiasis (HSC) is a rare - 1 but severe complication of long-lasting chemotherapy observed in immuno-compromised patients with prolonged neutropenia. It is considered as the most frequent form of chronic disseminated candidiasis. It typically occurs among patients with long-term neutropenia defined by more than 10 days of absolute neutrophil counts (ANC) below 0,5 G/L, occurring after induction of chemotherapy for acute leukemia [1]. According to Cornely et al., fever beginning at the time of ANC recovery and persisting in a previously afebrile patient should prompt an investigation for hepato-splenic candidiasis [2]. In this condition, blood cultures often evidence no fungi [3]. Liver biopsies are commonly performed, but histology and fungal cultures are often negative.

We report a case of severe refractory hepato-

$\overline{\text { Corresponding author }}$

Alexandre Peinoit

E-mail: a.peinoit@rennes.unicancer.fr splenic candidiasis confirmed after a splenectomy in a 54-year-old woman treated for acute lymphoblastic leukemia.

\section{CASE PRESENTATION}

A 54-year-old female patient was admitted to our hematology department for the treatment of B-cell acute lymphoblastic leukemia in August 2019. She had a medical history of localized left breast cancer diagnosed in March 2018 treated by mastectomy and axillary curettage, adjuvant chemotherapy with cyclophosphamide, epirubicin and paclitaxel, radiotherapy, and letrozole hormone therapy. The diagnosis of B-cell acute lymphoblastic leukemia was reached in a context of asthenia and NYHA class II dyspnea experienced since mid-August 2019 and associated with skin and mucosal hemorrhagic syndrome.

The initial blood tests showed severe anemia (hemoglobin: $5.3 \mathrm{~g} / \mathrm{dL}, \mathrm{MCV}: 101 \mathrm{fL}$ ), thrombocytopenia (platelets: $16 \mathrm{G} / \mathrm{L}$ ), and elevated white blood-cell count with blasts (total: $91.1 \mathrm{G} / \mathrm{L}$, including neutrophils: $2.73 \mathrm{G} / \mathrm{L}$, basophils: 0.91 G/L, lymphocytes: $1.82 \mathrm{G} / \mathrm{L}$ and blast cells: 84.7 
G/L). There was no electrolyte disturbance. Tests showed increased LDH levels (593 IU/L) with a normal liver test. The cerebral spinal fluid was cytologically normal. Karyotyping showed a translocation $\mathrm{t}(4 ; 11)$ leading to an aberrant MLL-AF4 transcription. The patient was included in the French GRAALL protocol (NCT0032767). She received induction chemotherapy including daunorubicin $150 \mathrm{mg} / \mathrm{m}^{2}$ (250 $\left.\mathrm{mg}\right)$, cyclophosphamide $1500 \mathrm{mg} / \mathrm{m}^{2}$ (2440 mg), vincristine $2 \mathrm{mg} / \mathrm{m}^{2}(8$ $\mathrm{mg}$ ), and L-asparaginase $36000 \mathrm{IU} / \mathrm{m}^{2}$ (58 680 IU), leading to prolonged neutropenia (29 days). The first febrile episode began after 13 days of aplasia. We considered the first day of fever as day 0 of the infection for this case report. Fever persisted with daily peaks, which were mainly vesperal and nocturnal, over 3 weeks. Blood tests showed cytolysis with maximum levels of AST: 102 IU/L (upper value: 31 IU/L), ALT: 293 IU/L (upper value: $34 \mathrm{IU} / \mathrm{L}$ ) at day 48 and cholestasis (GGT: $581 \mathrm{IU} / \mathrm{L}$, upper value: $38 \mathrm{IU} / \mathrm{L}$, and ALP: $650 \mathrm{IU} / \mathrm{L}$, upper value $105 \mathrm{IU} / \mathrm{L}$ ).

\section{Radiological findings}

An abdominal CT scan performed on day 12 showed multiple hypo-dense spleen nodules. First, an 18F-FDG PET/CT scan performed on day 29 evidenced multiples hypermetabolics spots in the liver and spleen, suggestive of hepato-splenic candidiasis (Figure 1).

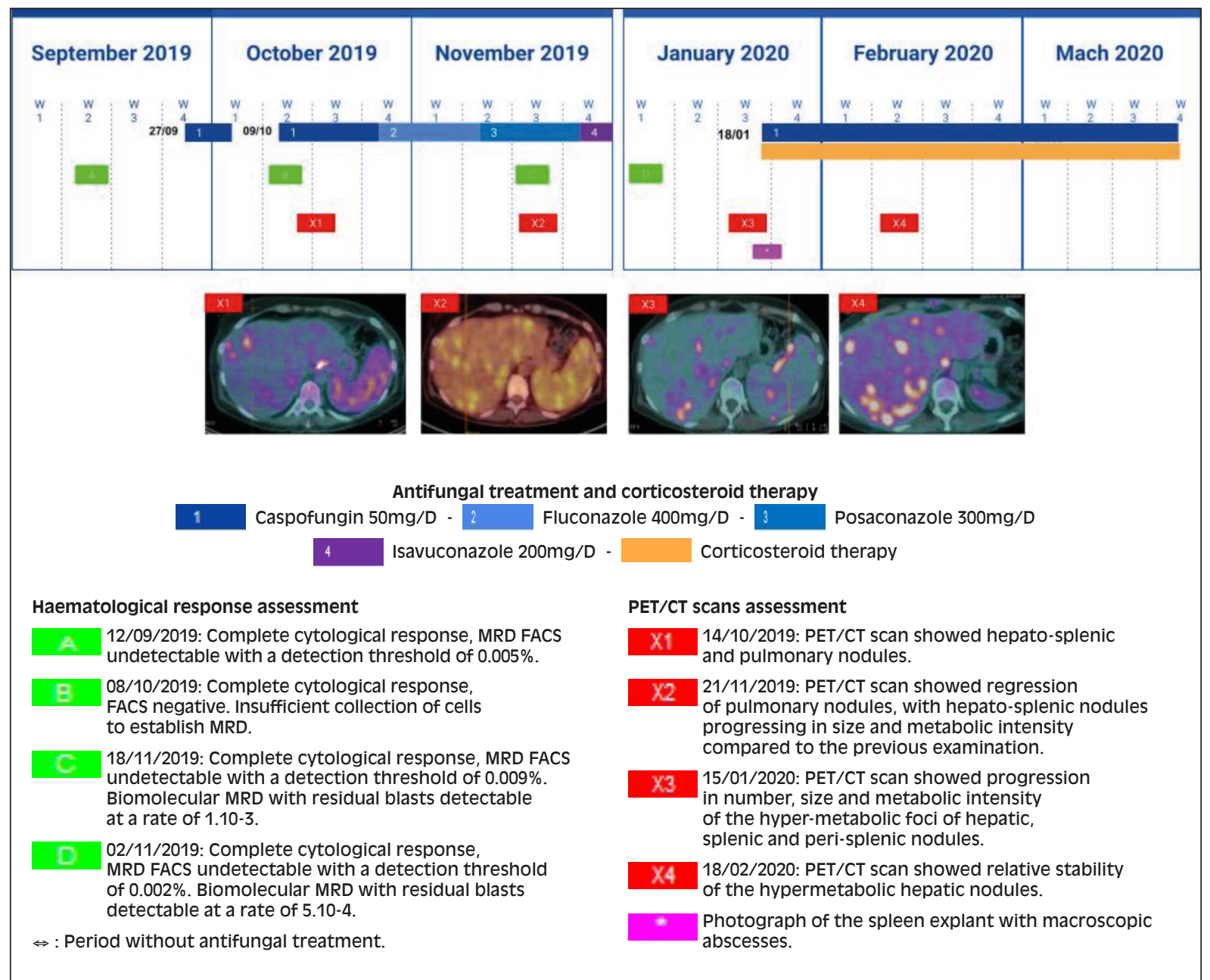

Figure 1 - Graphic representation of the evolution of care provision in the biological, radiological and therapeutic spheres. 


\section{Biological tests}

Bacteriological and fungal blood cultures, B-D glucan assay, 16S RNA blood viral assay, and bacterial, parasitological and mycological PCR assays failed to identify any microorganism. CA 15.3 and CA 19.9 values were normal.

All parasites and fungi (Aspergillus, Cryptococcus, Candida, Distomatosis, Toxocara, Echinococcus, Toxoplasma, Mucor, Rhizomucor, Lichtheimia) were tested negative. Only one Candida serology was positive at $65 \mathrm{AU} / \mathrm{L}$, signaling yeast contact (positivity threshold $\geq 10$ AU). The cultures of the explanted spleen were negatives. The viral PCRs were also negative.

We collected in total 75 bacterial blood cultures, 45 fungal blood cultures, two Candida serologies, one Mucor, Rhizomucor, Lichtheimia PCR, and three Beta-D-glucan.

\section{Pathological analyses}

The patient underwent two image-guided liver biopsies on days 32 and 44, and one surgical liver biopsy on day 79. Microbiological analysis of the three specimens did not evidence any causative microorganism.

On day 124, the patient underwent a splenectomy because of increased liver damage and persistent fever (Figure 2). Pathological examination of the spleen excluded a relapse of LAL, and evidenced splenic candidiasis with abscesses and epithelioids granulomas. No fungus was isolated from the spleen specimen cultures.

\section{Clinical outcome}

The patient exhibited complete cytological response after the induction phase. A follow-up of minimal residual disease (MRD) is provided in Figure 1.

In view of the exclusion of other causes of fever and the lack of improvement after antibiotics, 4 consecutive lines of antifungal treatment were administered: caspofungin $50 \mathrm{mg} /$ day from day 12 to day 19 and from day 24 to day 39, fluconazole $400 \mathrm{mg}$ / day from day 39 to day 52, posaconazole $300 \mathrm{mg} /$ day from day 52 to day 65 and isavuconazole $400 \mathrm{mg}$ / day from day 65 to day 68 .

The liver biopsies contributed no further information. Furthermore, the lack of improvement after several lines of antifungal treatment motivated a splenectomy, which finally confirmed the diagnosis of splenic candidiasis. Caspofungin was resumed and associated with steroids on day 125

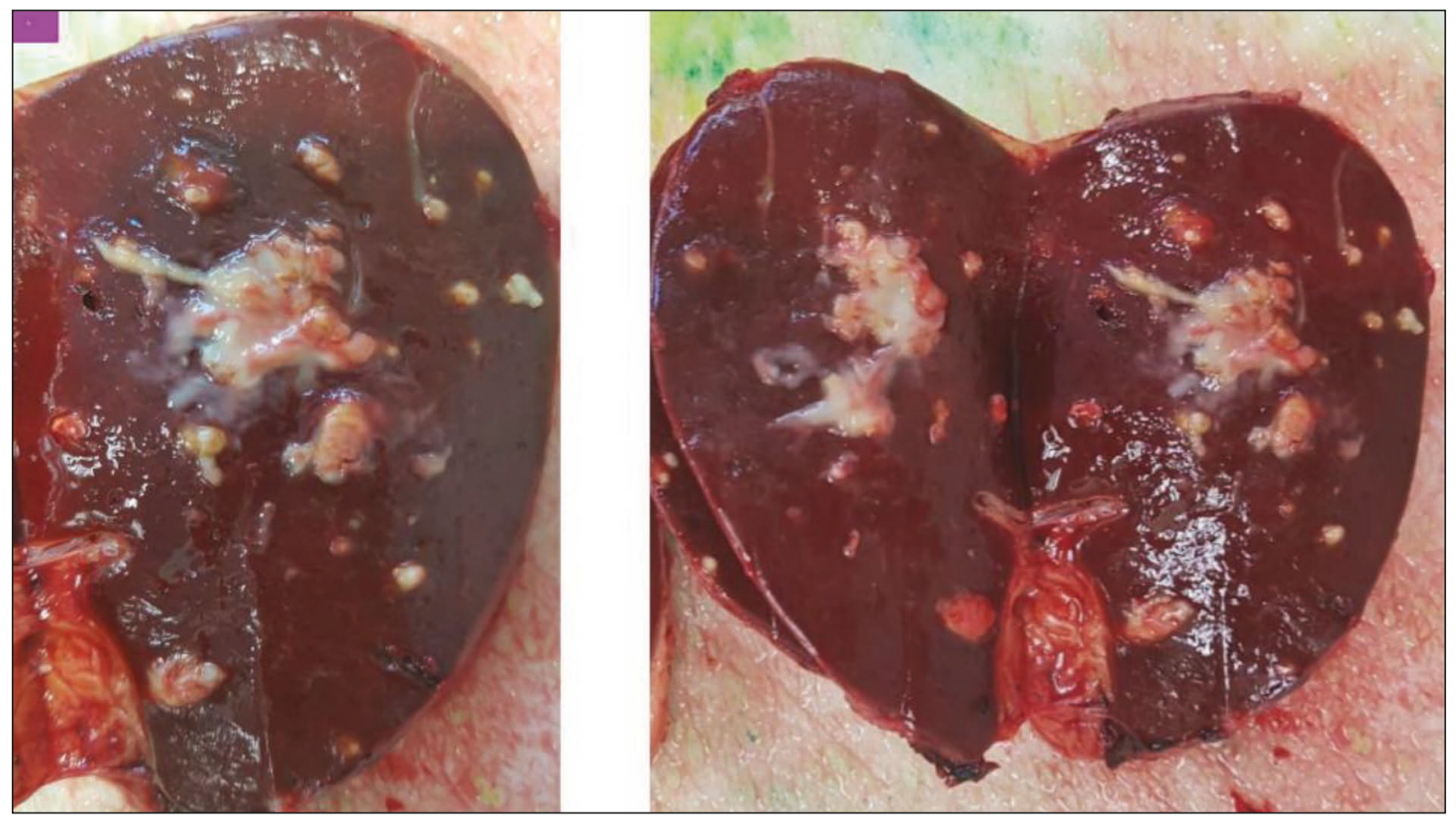

Figure 2 - Macroscopic anatomo-pathological images of the explanted spleen. 
for 3 weeks, resulting in apyrexia. Despite the recurrence of fever after steroid discontinuation, successive PET/CT scans showed metabolic stabilization of the lesions (Figure 1). Caspofungin was maintained for several months and was followed up with Amphotericin B combined with Flucytosine at day 429 for 2 weeks. The patient is currently treated with Amphotericin B. Repeated PET/CT scans have evidenced metabolic stabilization of the hepatic nodules.

The change of antifungal agent was operated in front of the lack of clinical improvement and on the advice of the infectious diseases specialits, then the antifungal agents were stopped in the assumption that it was not an invasive fungal infection.

The patient is still under treatment with long-term antifungal medication and is monitored by infectious diseases specialists on a regular basis, several times a month. Biological monitoring, particularly hematological, hepatic and Beta-D-Glucan, is also performed. This monitoring and follow-up is adjusted according to the overall evolution.

\section{DISCUSSION}

We report a case of severe hepato-splenic candidiasis in a 54-year-old woman treated for acute lymphoblastic leukemia. The diagnosis of hepatosplenic candidiasis is difficult to establish. Liver biopsies are rarely helpful to confirm hepatosplenic candidiasis, since fungal tissue invasion is difficult to prove because of the small sample sizes. In addition, the pathological syndrome is a combination of fungal abscesses and immune reconstitution inflammatory syndrome. The authors recommend starting treatment even without histological confirmation in case of high clinical probability of hepato-splenic candidiasis [2]. In the present case, all successive liver biopsies were considered negative, and the diagnosis was made on the basis of a resected spleen analysis. Antifungal treatment should be started without delay and the diagnostic procedure should not have an impact on leukemia management. Further clinical case presentations could improve clinicians' knowledge and diagnostic procedures for hepato-splenic candidiasis.

Imaging procedures are highly recommended in hepato-splenic candidiasis, especially when the microbiological diagnosis is not established. MRI is the most sensitive technique for an accurate diagnosis of hepato-splenic candidiasis, while CT scan and ultrasonography have lower sensitivity [4]. The actual role of 18F-FDG PET/CT scan in evaluating hepato-splenic candidiasis remains unclear. In the literature, some reports have demonstrated FDG uptake in hepato-splenic candidiasis and underlined the benefit for the diagnosis of hepato-splenic infection [5, 6]. Because inflammatory lesions persist for a long time around hepatic and splenic abscesses in candidiasis even after fungus sterilization, the role of PET/CT scan in the follow-up of hepato-splenic candidiasis needs to be studied.

The recent guidelines issued by the European Society of Clinical Microbiology and Infectious Diseases recommend fluconazole as the first-line treatment for the microorganisms most commonly isolated from biopsy specimens (i.e., C. albicans, C. tropicalis, and C. parapsilosis). Fluconazole at a daily dose of $400 \mathrm{mg}$ is recommended as a first-line treatment for at least 3 months, concomitantly with prednisone at doses of $\geq 0.5 \mathrm{mg}$ / $\mathrm{kg}$ for at least 3 weeks. Liposomal amphotericin $\mathrm{B}$ and echinocandin antifungals are considered as alternative therapies [7]. In this case, we used caspofungin at a daily dose of $50 \mathrm{mg}$ on account of increased hepatic lesions observed after treatment with fluconazole, then followed up with amphotericin B.

Splenectomy is rarely performed in case of suspected hepato-splenic candidiasis. In the first case reported by Raina et al., hepato-splenic candidiasis was confirmed after a splenectomy performed in the context of hyper-splenism in a patient treated for Hodgkin's disease. A histopathological examination of the spleen showed fungal granulomas of Candida albicans [8]. Migita et al. reported one case of multiple fungal splenic abscesses in a patient with T-acute lymphoblastic leukemia, undergoing chemotherapy. The histological study of the resected spleen showed multiple granuloma formations and microscope examination showed budding ovoid yeast cells on Grocott staining, that were strongly compatible with Candida. All the serological analyses were negative in this case [9].

According to the criteria, the diagnosis of hepatosplenic candidiasis in immuno-compromised patients is considered as proven when pathological examinations of biological samples evidence the 
presence of fungus. In this case, since the liver biopsies were not contributive, and several lines of antifungal treatments did not induce any clinical benefit, it was the splenectomy that confirmed the hepato-splenic diagnosis, and eventually guided the choice of antifungal agents [10].

\section{CONCLUSIONS}

Hepato-splenic candidiasis is a very rare disease and difficult to diagnose in immuno-compromised patients. Liver biopsies are frequently performed, but the histology and fungal cultures are often negative. Splenectomy can be helpful in proving fungal infection, in excluding relapse, and in guiding the choice of antifungal agents.

\section{Conflict of interest}

No conflict of interest to disclose.

\section{Funding}

The authors did not receive support from any organization for the submitted work.

\section{Ethics approval}

The direction of research and ethics has confirmed that no ethical approval is required.

\section{Consent to participate and to publish}

Written informed consent was obtained from the patient.

The participant has consented to the submission of the case report to the journal.

\section{Authorship}

Alexandre Peinoit, Elie Cousin, and Thierry Lamy carried out the research and wrote the paper.

Martine Escoffre-Barble, Marc Bernard, and François Benezit provided substantial data for the research study.

All the authors substantially contributed to revising the manuscript, they all read and approved the final version.

\section{Acknowledgements}

We would like to thank the patient who did not object to the disclosure of her anonymized data.

\section{REFERENCES}

[1] Pagano L, Mele L, Fianchi L, et al. Chronic disseminated candidiasis in patients with hematologic malignancies. Clinical features and outcome of 29 episodes. Haematologica. 2002; 87 (5), 535-41.

[2] Cornely OA, Bangard C, Jaspers NI. Hepatosplenic candidiasis. Clin Liver Dis. 2015; 6 (2), 47-50.

[3] Rammaert B, Desjardins A, Lortholary O. New insights into hepatosplenic candidosis, a manifestation of chronic disseminated candidosis. Mycoses. 2012; 55 (3), 74-84.

[4] Masood A, Sallah S. Chronic disseminated candidiasis in patients with acute leukemia: emphasis on diagnostic definition and treatment. Leuk Res. 2005; 29 (5), 493-501.

[5] Hot A, Maunoury C, Poiree S, et al. Diagnostic contribution of positron emission tomography with [18F] fluorodeoxyglucose for invasive fungal infections. Clin Microbiol Infect. 2011; 17 (3), 409-17.

[6] Teyton P, Baillet G, Hindié E, et al. Hepatosplenic Candidiasis Imaged With F-18 FDG PET/CT. Clin Nucl Med. 2009; 34 (7), 439-40.

[7] Ullmann AJ, Akova M, Herbrecht R, et al. Guideline for the diagnosis and management of Candida diseases 2012: adults with haematological malignancies and after haematopoietic stem cell transplantation (HCT). European Society for Clinical Microbiology and Infectious Diseases. Clin Microbiol Infect. 2012; (Suppl. 7), 53-67.

[8] Raina V, Young PT, Foulis AK, Soukop M. Hypersplenism due to fungal infection of spleen in a successfully treated patient with Hodgkin's disease. Postgrad Med J. 1989; 65 (760), 83-5.

[9] Migita M, Kaizu K, Asai M, et al. Multiple fungal splenic abscesses in a patient with T-acute lymphoblastic leukemia undergoing chemotherapy. Pediatr Int. 2004; 46 (6), 733-5.

[10] Donnelly JP, Chen SC, Kauffman CA, et al. Revision and update of the consensus definitions of invasive fungal disease from the European organization for research and treatment of cancer and the mycoses study group education and research consortium. Clin Infect Dis. 2020; 71 (6), 1367-13. 